\title{
Decision makers need an approach to determine digital therapeutic product quality, access, and appropriate use
}

\author{
Benjamin Parcher, PharmD, MS, and Megan Coder, PharmD
}

As clinicians and managed care decision makers increasingly encounter and review digital therapeutics (DTx) for use in patient care, it is critical for industry stakeholders to develop a harmonized approach to DTx evaluation. Building on the maturation of the digital therapeutic class from an industry and regulatory standpoint, ${ }^{1,2}$ this commentary recommends considerations related to how DTx may be evaluated from a clinical standpoint and subsequently adopted into clinical practice for target patient populations.

Our health care system, and society in general, has been fundamentally and perhaps inalterably changed by COVID-19. While it is still unclear what the new normal may be, it is becoming increasingly clear across the managed care spectrum that alternative and novel means to deliver nonemergent care is a mission-critical objective. The rapid adoption of telemedicine in recent months represents a paradigm shift created, or at least accelerated, by the pandemic. But while helpful, telemedicine has inherent limitations for delivering therapeutic benefits in the current environment because it requires the availability and time of health care professionals to enable and deliver care, albeit remotely.

As the digital frontier has expanded over the past decade, available technologies have evolved from wellness apps and telehealth platforms, to monitoring and diagnostic products, and now to digital therapeutics, which use software to directly deliver clinical interventions to patients. AMCP has actively supported efforts to properly assess the value DTx products offer to patients and caregivers and strengthen patient-clinician relationships through the provision of actionable product-generated insights that directly affect health care decisions. ${ }^{2}$

DTx is defined by AMCP as software that delivers a clinical mechanism of action, either alone or in combination with other standard-of-care treatments to improve outcomes. ${ }^{3}$

Since DTx product quality, access, and appropriate use are so critical to health care decision makers, it is important that patients, clinicians, and payers can identify, assess, and meaningfully use DTx for the purpose of treating, managing, and preventing the diseases and disorders in which they are used. Here, we provide a general approach for evaluating DTx products.

\section{Product Claims Matter}

Across the spectrum of digital health, there are significant differences between product types, intended uses, levels of clinical evidence, and required levels of regulatory oversight. Since end users may not be able to fully evaluate all of these factors before product use, health care decision makers (HCDM) serve as critical conduits in this assessment process.

\author{
Author affiliations \\ Benjamin Parcher, PharmD, MS, Xcenda, \\ AmerisourceBergen, Palm Harbor, FL, \\ and Megan Coder, PharmD, Digital \\ Therapeutics Alliance, Arlington, VA. \\ AUTHOR CORRESPONDENCE: \\ Benjamin Parcher, \\ bparcher@gmail.com \\ J Manag Care Spec Pharm. \\ 2021;27(4):536-38 \\ Copyright $\odot 2021$, Academy of Managed \\ Care Pharmacy. All rights reserved.
}

Depending on the desired outcome-whether patient mood improvement, improved insight on patient daily progress for clinicians, or remotely delivered treatments for depression or insomnia-it is important to select the appropriate product, such as a wellness app, remote monitoring tool or sensor, or DTx.

\section{Product Quality Matters}

In evaluating product quality, it is important for HCDMs to know that general digital health products do not require clinical evidence or regulatory oversight, since they do not make 
medical claims. In contrast, DTx must adhere to each of the follow industry standards ${ }^{4}$ :

- Prevent, manage, or treat a medical disorder or disease

- Produce a medical intervention that is driven by software

- Incorporate design, manufacture, and quality best practices

- Engage end users in product development and usability processes

- Incorporate patient privacy and security protections

- Apply product deployment, management, and maintenance best practices

- Publish trial results inclusive of clinically meaningful outcomes in peer-reviewed journals

- Be reviewed and cleared or approved by regulatory bodies as required to support product claims of risk, efficacy, and intended use (e.g., FDA clearance)

- Make claims appropriate to clinical validation and regulatory status

- Collect, analyze, and apply real-world evidence and/or product performance data

Whether DTx products are used as standalone therapies or in concert with medications, devices, or other therapies to optimize patient care, HCDMs may apply industry patient-centered core principles, ${ }^{5}$ ethical standards, ${ }^{6}$ and product development best practices ${ }^{7}$ to the DTx to verify product integrity, user-centered designs, patient privacy, and validated clinical outcomes.

\section{DTx Payor Policies}

While the debate continues regarding what insurance benefit category DTx should fall under (e.g., medical, pharmacy, or other pathway), there is already a handful of published payer policies that offer a glimpse into how DTx products are being evaluated and playing a role in therapy.

One example that offers a glimpse into how the coverage policy landscape is evolving is that of the newly approved DTx platform for attention-deficit/hyperactivity disorder (ADHD), EndeavorRx (AKL-T01) by Akili Interactive. ${ }^{8}$ As of its U.S. Food and Drug Administration (FDA) approval date (June 15, 2020), several prominent managed care organizations $(\mathrm{MCO})$ had already cited the platform on their respective coverage polices for ADHD.

In one of these policies, a large MCO had listed Akili's EndeavorRx as experimental/investigational under a blanket status for the category termed "brain training/ cognitive programs/games, ${ }^{9}$ While one might contend that this is an appropriate status, since the policy review date preceded the product's approval date, the policy's review was nevertheless subsequent to data available from clinical studies that demonstrated the platform's benefit in children diagnosed with ADHD. Rather than cite these studies specifically, the policy grouped Akili's platform with other brain training/cognitive programs/games platforms that held considerably less clinical evidence to substantiate their benefit. Moreover, studies that were cited included meta-analyses published in 2013-2014 that looked at even earlier platforms in their prototypic state. ${ }^{10,11}$ One would hope that EndeavorRx's recent FDA approval would prompt a dedicated look at its published evidence and encourage a discussion that would distinguish it from wellness products and technologies and publications from over 10 years ago.

\section{Appropriate Use Is Crucial}

Subsequent to assessments of DTx quality, clinical evidence, and regulatory oversight, HCDMs must determine how they intend to use each product. Given DTx product requirements for clinician, payer, or employer authorization, the following "rights" will help guide appropriate product use and ensure that the right care is delivered at the right time.

Considerations related to initial clinical care include the following:

- The right indication: Which disease or disorder does the product have an indication to treat, manage, or prevent?

- The right patient: For which patients does this product offer targeted, evidence-based, clinically evaluated therapies?

- The right therapy: Does this product align with the patient's current needs and care pathway? Should this product be used independently? In tandem with remote or in-person clinician-delivered therapy? Paired with a medication, device (e.g., sensor, glucometer), or other therapy?

- The right timing and frequency: What are the timing, duration, frequency, and target termination of therapy use?

- The right cultural references: Is product content available to the patient in a familiar language and with appropriate cultural references?

Considerations related to ongoing care assessment include the following:

- The right outcomes documentation: What clinical outcomes are collected by the product and shared with patients, caregivers, and clinicians?

- The right response to therapy: How are real-world outcomes leveraged to detect adverse events and nonoptimal outcomes, in addition to assessing product utilization, engagement, and success of therapy? 
- The right evaluation: How are actionable insights leveraged by clinicians to assess and optimize overall therapy?

DTx are designed to meet specific patient needs. They align with clinical guidelines, integrate into health systems, and are used in the context of patients' current care plans. It is therefore crucial that appropriate product use is ensured once access to high-quality DTx have been provided.

\section{Conclusions}

DTx create new treatment and selfmanagement options for patients with a broad range of behavioral, mental, and physical diseases and disorders. Building on the ease of product scalability and access through patientowned devices, DTx products can more easily reach high-risk, rural, and underserved communities that often lack access to health care services even during the best of times.

As this emerging category of medicine continues to evolve, it is important for health care decision makers to understand (a) the differences between digital wellness, monitoring, diagnostic, adherence, and therapeutics products; (b) how to assess the quality of products, depending on their level of risk and intended use; (c) how to develop benefit category assignments for DTx products, given their FDA recognition as medical devices and parallel ability to perform in line with traditional pharmaceutical therapies; and (d) the value that DTx provide to patients when used in the appropriate context of care.

\section{DISCLOSURES}

No funding was received for the writing of this article. Parcher is employed by Xcenda and has nothing to disclose. Coder is employed by Digital Therapeutics Alliance, which is a member of the USP Convention. Coder reports fees from PsychU/Otsuka.

\section{REFERENCES}

1. U.S. Food and Drug Administration. Guidances with digital health content. Updated October 6, 2020. Accessed March 6, 2021. https://www.fda. gov/medical-devices/digital-health/ guidances-digital-health-content

2. Digital Therapeutics Alliance. About DTA. January 1, 2020. Accessed March 6, 2021. https://dtxalliance.org/about-dta/

3. AMCP Partnership Forum: Digital therapeutics-what are they and where do they fit in pharmacy and medical benefits? J Manag Care Spec Pharm. 2020;26(5):674-81. doi: 10.18553/ jmcp.2020.19418.

4. Digital Therapeutics Alliance. Ensuring appropriate quality, access, and utilization of digital therapeutics. Fact sheet. April 2020. Accessed March 6, 2021. https://dtxalliance.org/wp-content/ uploads/2020/04/DTx Quality Access Utilization Worksheet.pdf

5. Digital Therapeutics Alliance. Digital therapeutics definition and core principles. Fact sheet. November 2019. Accessed March 6, 2021. https://dtxalliance.org/ wp-content/uploads/2019/11/DTA_DTxDefinition-and-Core-Principles.pdf
6. Digital Therapeutics Alliance. DTx industry code of ethics. Fact sheet. November 2019. Accessed March 6, 2021. https://dtxalliance.org/wp-content/ uploads/2019/11/DTA_DTx-IndustryCode-of-Ethics_11.11.19.pdf

7. Digital Therapeutics Alliance. DTx product best practices. Fact sheet. November 2019. Accessed March 6, 2021. https://dtxalliance.org/wp-content/ uploads/2019/11/DTA_DTx-ProductBest-Practices_11.11.19.pdf

8. Akili. Akili announces FDA clearance of EndeavorRx ${ }^{\mathrm{TM}}$ for children with ADHD, the first prescription treatment delivered through a video game. Press release. June 15, 2020. Accessed March 6, 2021. https://www.akiliinteractive.com/ news-collection/akili-announces-endeavortm-attention-treatment-is-now-available-for-children-with-attention-deficithyperactivity-disorder-adhd-al3pw

9. Cigna. Attention-deficit/hyperactivity disorder (ADHD): assessment and treatment. Medical coverage policy: 0231. January 15, 2020. Accessed March 6, 2021. https://static.cigna.com/assets/ chcp/pdf/coveragePolicies/medical/ $\mathrm{mm} 0231$ coveragepositioncriteria adhd_assessment_and_treatment.pdf

10. Rapport MD, Orban SA, Kofler MJ, Friedman LM. Do programs designed to train working memory, other executive functions, and attention benefit children with ADHD? A meta-analytic review of cognitive, academic, and behavioral outcomes. Clin Psychol Rev. 2013;33(8):1237-52.

11. Sonuga-Barke E, Brandeis D, Holtmann M, Cortese S. Computer-based cognitive training for ADHD: a review of current evidence. Child Adolesc Psychiatr Clin N Am. 2014;23(4):807-24. 